\title{
Elizabethkingia meningoseptica causing meningitis after decompressive craniectomy for traumatic brain injury in a immunocompetent adult: Serious Neurocritical care Concern
}

\author{
Guru Dutta Satyarthee, Mayank Garg, Deepak Agarwal \\ Department of Neurosurgery, All India Institute of medical Sciences, New Delhi, INDIA
}

\begin{abstract}
Elizabethkingia is aerobic Gram negative bacteria is widely distributed in soil, food and food. It is very rarely pathogenic for human and especially those who are immunocompromised and various risk factors includes intravenous catheter, ventilatory support. We report a case, who had undergone decompressive craniectomy for traumatic brain injury, developed meningitis, CSF culture showed growth of Elizabethkingia and treated successfully with antibiotics therapy. This study reminds that rare pathogen should also be considered as causative organism for meningitis especially in postoperative cases and it responds well to antimicrobial therapy.

Key words: Meningitis, post-decompressive surgery, immunocompetent host, treatment
\end{abstract}

\section{Introduction}

Elizabethkingia

meningoseptica, previously termed as Flavobacterium meningosepticum or Chryseobacterium meningosepticum is an aerobic Gramnegative, rod-shaped bacterium (15).

It is a rare human pathogen, widely distributed in soil, plants, food and potable water and wet surface in the hospital environment. It can cause serious infections in immunosupressed and intubated patients with prolonged mechanical ventilation. Intravascular devices such as intravenous catheter, urinary catheter, intravascular implant are important risk factors.

Historically, it was associated with neonatal meningitis and sepsis, first identified by King Elizabeth in 1959 (7).

Subsequently, outbreaks of neonatal meningitis, septicaemia and pneumonia in severely immunocompromised and prolonged ventilated individuals were reported. E. meningoseptica is considered inherently resistant to the usual empiric therapy aimed at Gram-negative bacilli. Authors report a rare case of meningitis caused by E. meningoseptica in an adult operated for severe head injury, managed at tertiary care trauma centre. 


\section{Case illustration}

A 26- year-old patient was admitted with acute subdural haemorrhage following road traffic accident with Glasgow coma scale of E1VtM2 and underwent decompressive craniectomy and discharged from hospital with GCS of E1VtM3 status. Two months following discharge, he was readmitted with high grade fever. On admission vitals were stable with tense bulging flap on decompressive craniectomy site and neck rigidity. Routine urinary examination, haematological and biochemistry tests were carried out. Repeat computed cranial tomography scan revealed presence of presence of hydrocephalus associated with gross dilatation of ventricles. An emergency CSF diversionary procedure, external ventricular drain placement was performed. CSF sample sent for bacterial cultures identified the isolated bacterium as gram negative bacilli, E. meningoseptica.

CSF sugar level was $30 \mathrm{mg} / \mathrm{dl}$ and CSF protein was $240 \mathrm{mg} / \mathrm{dl}$. The isolate was sensitive to piperacillin-tazobactam, cotrimoxazole, tigecycline and vancomycin. Patient was treated with intravenous antibiotics according to culture sensitivity for three weeks, to which he responded favourably.

\section{Discussion}

E. meningoseptica, is a non-fermentor Gram-negative bacillus, widely found in water, soil and wet surfaces of the hospital environment. Environmental studies have indicated that this organism can survive even in chlorine-treated water, colonizes in sink basins and taps and ventilator tubing (15).

Most of the reported cases are of neonatal meningitis (7). Isolation of this organism in adults cases of pneumonia, endocarditis and meningitis are reported but none in a case of severe head injury (3).

Bloch et al (2) reported in 1997 a series of 15 immunocompromised patients with $\mathrm{E}$. meningosepticum infections. $80 \%$ of the patients had nosoconmial-acquired infections while $20 \%$ were colonized with E. meningosepticum without evidence of infection.

The incidence of E. meningoseptica may be under-reported as identification is difficult without an automated system. Environmental samples should be taken to identify the source of infection. Treatment of E. meningoseptica infection is challenging because of organism's multidrug resistance. Quinolones, vancomycin, trimethoprim-sulfamethoxazole, and rifampicin have been documented as potential therapies (5). E. meningoseptica is usually resistant to colistin and carbapenems which are used to treat extended-spectrum beta-lactamase-producing Klebsiella pneumoniae and Acinetobacter baumannii, and these characteristics give it a survival advantage.

The role of tigecycline is not defined, but our patient had a clinical response after treatment with tigecyclin, as the culture showed sensitivity to it. Shailja et al (12) showed that E. meningoseptica isolates were resistant to cephalosporins, aminoglycosides, trimethoprim-sulfamethoxazole, beta -lactam combinations, carbapenems while only one 
isolate was susceptible to ciprofloxacin. All the isolates were susceptible to vancomycin, still six of nine neonates died even after using vancomycin. Though the organism is susceptible to vancomycin in vitro, its efficacy in vivo is questionable.

About ten cases of adult meningitis are reported in literature $(1,3,4,8-11,13-15)$ but only two cases after neurosurgical procedure (Table 1). This is probably the first case report of E.meningoseptica causing meningitis in trauma patients. Severe head injury leads to prolonged hospital stay of these predominantly young adult patients who otherwise have no other co-morbidities. High degree of suspicion with rapid diagnosis and prompt institution of appropriate therapy for prolonged period (about 3-4 weeks) are essential in the management of such infections.

TABLE 1

Review of important cases developing meningitis following neurosurgical procedure

\begin{tabular}{|l|l|l|l|l|l|}
\hline S. no. & Author/ reference & year & $\begin{array}{l}\text { Preceding neurosurgical } \\
\text { procedure }\end{array}$ & $\begin{array}{l}\text { Interval of } \\
\text { meningitis onset }\end{array}$ & outcome \\
\hline 1 & Wang et al (14) & 2014 & $\begin{array}{l}\text { Aneurysm clipping in } \\
73 \text {-year old male }\end{array}$ & 10 days & favourable \\
\hline 2 & Chan et al (3) & 1983 & $\begin{array}{l}\text { Transsphenoidal } \\
\text { hypophysectomy }\end{array}$ & Not reported & favourable \\
\hline 3 & Current case & 2017 & $\begin{array}{l}\text { Decompressive } \\
\text { craniectomy }\end{array}$ & Two- months & Excellent \\
\hline
\end{tabular}

About $90 \%$ of reported cases of adult meningitis have debilitating predisposing conditions ie. hematological disorders, immunosuppressive state, neoplasms and diabetes mellitus. The common manifestations included fever, disturbances of consciousness, seizure, hydrocephalus and stroke. The initial CSF cultures and Gram stains were inconclusive in 50\% cases. Despite treatment, about $50 \%$ died during the course of treatment (9).

$\mathrm{Lu}$ et al. observed the clinical and laboratory manifestations in the adult is similar to bacterial meningitis caused by other bacterium. So, clinical diagnosis may be confirmed by isolating positive CSF culture, for which repeated CSF cultures may be needed (9). Lu et al. reported a 21 year old diabetic patient with meningitis, confirmed by a positive CSF culture. She presented with fever, headache, consciousness disturbance, and seizure. CSF analysis revealed a purulent inflammatory reaction. She was discharged after 21-day course of intravenous antibiotics therapy (9).

Wang et al. (14) reported a 73-year-old male patient of ruptured middle cerebral artery aneurysm which was clipped followed by lumbar external drainage for acute hydrocephalus. Ten days later, CSF culture showed growth of E. meningoseptica which responded favourably. 
Chan et al (3) in 1983 reviewed literature of Flavobacterium meningosepticum causing adult meningitis and could find only five cases. Chan et al reported a female developing meningitis following transsphenoidal hypophysectomy surgery. She recovered completely with oral rifampicin and intravenous chloramphenicol and cefoperazone.

Jean et al. (6) observed Elizabethkingia meningoseptica as a potential threat to patients in critical care unit because of multidrug resistance which may produce $\mathrm{E}$. meningoseptica outbreaks and create an important health issue. Control of such outbreak requires strictly following standard infection control measures, isolation of contact, identification of the source and very important is cleaning of equipment.

\section{Conclusion}

Elizabethkingia meningoseptica possess a potentially important threat to patients in neurosurgical critical care unit due to existence of multidrug resistance causing E. meningoseptica outbreaks. It is highly important laboratories should have the facilities to identify the organism. Awareness about this organism and sensitivity testing is required to reduce morbidity and control outbreaks. Authors presented case of Elizabethkingia meningoseptica causing meningitis in an operated case of decompressive craniectomy for traumatic brain injury with successful treatment.

\section{Correspondence}

Dr. Guru Dutta Satyarthee

Associate Professor
Department of Neurosurgery

CNC building, AIIMS

Ansari nagar, New Delhi, India - 110029

Email:duttaguru2002@yahoo.com

Phone no. : +919868398243

\section{References}

1.Bagely DHJ, Alexander JCJ, Gill VJ, Dolin R, Ketcham AS. Late Flavobacterium species meningitis after craniofacial exenteration. Arch Intern Med. 1976 Feb; 136(2): p. 229-31.

2.Bloch KC, Nadarajah R, Jacobs R. Chryseobacterium meningosepticum: an emerging pathogen among immunocompromised adults. Report of 6 cases and literature review. Medicine (Baltimore). 1997 Jan; 76(1): p. 30-41.

3.Chan KH, Chau PY, Wang RY, Huang CY. Meningitis caused by Flavobacterium meningosepticum after transsphenoidal hypophysectomy with recovery. Surg Neurol. 1983 Oct; 20(4): p. 294-6.

4.Harrington SP, Perlino CA. Flavobacterium meningosepticum sepsis: disease due to bacteria with unusual antibiotic susceptibility. South Med J. 1981 Jun; 74(6): p. 764-6.

5.Hung PP, Lin YH, Lin CF, Liu MF, Shi ZY. Chryseobacterium meningosepticum infection: antibiotic susceptibility and risk factors for mortality. J Microbiol Immunol Infect. 2008 Apr; 41(2): p. 137-44.

6.Jean SS, Lee WS, Chen FL, Ou TY, Hsueh PR. Elizabethkingia meningoseptica: an important emerging pathogen causing healthcare-associated infections. J Hosp Infect. 2014 Apr; 86(4): p. 244-9. doi: 10.1016/j.jhin.2014.01.009. Epub 2014 Feb 25.

7.King EO. Studies on a group of previously unclassified bacteria associated with meningitis in infants. Am J Clin Pathol. 1959 Mar; 31(3): p. 241-7.

8.Lim LC, Low JA, Chan KM. Chryseobacterium meningosepticum (Flavobacterium meningosepticum)-a report of five cases in a local hospital. Ann Acad Med Singapore. 1999 Nov; 28(6): p. 858-60.

9.Lu CH, Huang CR, Tsai NW, Chang CS, Chuang YC, Lee PY, et al. An adult case of Chryseobacterium meningosepticum meningitis. Jpn J Infect Dis. 2004 Oct; 57(5): p. 214-5.

10.Madruga M, Zanon U, Pereira GM, Galvão AC. Meningitis caused by Flavobacterium meningosepticum. 
The first epidemic outbreak of meningitis in the newborn in South America. J Infect Dis. 1970 Mar; 121(3): p. 32830.

11.Rios I, Klimek JJ, Maderazo E, Quintiliani R. Flavobacterium meningosepticum meningitis: report of selected aspects. Antimicrob Agents Chemother. 1978 Sep; 14(3): p. 444-7.

12.Shailaja VV, Reddy AK, Alimelu M, Sadanand LN. Neonatal Meningitis by Multidrug Resistant Elizabethkingia meningosepticum Identified by $16 \mathrm{~S}$ Ribosomal RNA Gene Sequencing. Int J Pediatr. 2014. 2014;: p. 918907. doi: 10.1155/2014/918907. Epub 2014 Feb 9.

13.Uchihara T, Yokota T, Watabiki S, Ueki M, Miyake S, Tsukagoshi H. Flavobacterium meningosepticum meningitis in an adult. Am J Med. 1988 Nov; 85(5): p 738-9.

14.Wang X, Hu Z, Fan Y, Wang H. Chryseobacterium indologenes catheter-related meningitis in an elderly patient after intracranial aneurysm clipping surgery. Neurol Sci. 2014 Jan; 35(1): p. 113-5. doi: 10.1007/s10072013-1500-z. Epub 2013 Jul 17.

15.Weaver KN, Jones RC, Albright R, Thomas Y, Zambrano CH, Costello M, et al. Acute emergence of Elizabethkingia meningoseptica infection among mechanically ventilated patients in a long-term acute care facility. Infect Control Hosp Epidemiol. 2010 Jan; 31(1): p. 54-8. doi: $10.1086 / 649223$. 